\title{
A COMPARATIVE STUDY OF THE PERFORMANCES OF SOME ESTIMATORS OF LINEAR MODEL WITH FIXED AND STOCHASTIC REGRESSORS
}

K. AYINDE AND J. O. IYANIWURA

(Received 26, February 2007; Revision Accepted 25, July 2008)

ABSTRACT

In linear regression model, regressors are assumed fixed in repeated sampling. This assumption is not always satisfied especially in business, economics and social sciences. Consequently in this paper, effort is made to compare the performances of some estimators of linear model with autocorrelated error terms when normally distributed regressors are fixed (non - stochastic) with when they are stochastic. The estimators are the ordinary least square (OLS) estimator and four feasible generalized least estimators which are Cochrane Orcutt (CORC), Hidreth - Lu (HILU), Maximum Likelihood (ML), Maximum Likelihood Grid (MLGD) estimator. These estimators are compared using the finite properties of estimators' criteria namely; sum of biases, sum of variances and sum of the mean squared error of the estimated parameter of the model at different levels of autocorrelation and sample size through Monte - Carlo studies.

Results show that at each level of autocorrelation the estimated value of the criteria with stochastic regressor are much lesser than that of the fixed regressor for all the estimators except CORC when the sample size is small $(n=20)$ and the level of autocorrelation is very high $(\rho=0.9)$. More comparatively, it is observed that the same estimator(s) that is more efficient with fixed regressors is also more efficient with stochastic regressors except when the sample size is large $(n=80)$ and the level of autocorrelation is either low $(\rho=0.4)$ or high $(\rho=0.8)$. At these instances, the CORC / HILU estimator is more efficient with fixed regressors while the ML / MLGD estimator is more efficient with stochastic regressors.

KEYWORDS: Fixed Regressors, Stochastic Regressors, Linear Model, Autocorrelated error, OLS estimator, Feasible GLS estimators.

\section{INTRODUCTION}

One of the basic assumptions that are made about the regressors in linear regression model is that they are fixed in repeated sampling. This assumption is not always satisfied especially in business, economics and social sciences. This is because their regressors are often generated by stochastic process beyond their control. For instance, consider regressing daily bathing suit sales by a departmental store on the mean daily temperature. Certainly, the departmental store can not control daily temperature, so it would not be meaningful to think of repeated samples when temperature levels are the same from sample to sample (Fomby et. al, 1984). Authors like Neter and Wasserman (1974), Maddala (2002) have given situations and instances where these assumptions may be not be tenable and have also discussed their consequences on the Ordinary Least Square (OLS) estimator when used to estimate the model parameters. Graybill (1961), Sampson (1974), Fomby et.al (1984) and many others emphasized that if regressors are stochastic and independent of the error terms; the OLS estimator is unbiased and has minimum variance even though it is not Best Linear Unbiased Estimator (BLUE). When all the assumptions of the linear regression model hold except that the error terms are not homoscedastic (i.e. $\left.E\left(U U^{1}\right) \neq \sigma^{2} I_{n}\right)$ but are heteroscedastic (i.e. $E\left(U U^{1}\right)=\sigma^{2} \Omega$ ), the resulting model the Generalized Least Squares (GLS) Model. Aitken (1935) has shown that the GLS estimator $\beta$ of $\beta$ given as

$$
\hat{\beta}=\left(X^{1} \Omega^{-1} X\right)^{-1} X^{1} \Omega^{-1} Y
$$

is efficient among the class of linear unbiased estimators of $\beta$ with variance - covariance matrix of $\hat{\beta}$ given as

$$
V(\hat{\beta})=\sigma^{2}\left(X^{1} \Omega^{-1} X\right)^{-1}
$$

where $\Omega$ is assumed to be known. However, $\Omega$ is not always known, it is often estimated by $\Omega$ to have what is known as Feasible GLS estimator. Many consistent estimates of $\hat{\Omega}$ can be obtained (Fomby et. al, 1984).

With first order autocorrelated error terms (AR (1)), among the Feasible GLS estimators in literature are the Cochrane and Orcutt estimator (1949), Hildreth and Lu estimator (1960), Prais - Winsten estimator (1954), Thornton estimator (1982), Durbin estimator (1960), Theil's estimator (1971), the Maximum Likelihood estimator and the Maximum Likelihood Grid estimator (Beach and Mackinnon, 1978). Some of these estimators have now been incorporated into White's SHAZAM program (White, 1978) and the new version of the time series processor (TSP, 2005).

Consequently, effort is made in this paper to compare the performances of some of these estimators of linear model when normally distributed regressors are fixed (non stochastic) in repeated sampling with when they are stochastic.

\section{LITERATURE REVIEW}

The OLS estimator has been widely discussed to be unbiased but suffer efficiency in estimating the parameters of

K. Ayinde, Department of Pure and Applied Mathematics, Ladoke Akintola University of Technology, P. M. B. 4000, Ogbomoso, Oyo State, Nigeria.

J. O. Iyaniwura, Department of Mathematical Sciences, Olabisi Onabanjo University, P.M.B.2002, Ago-Iwoye, Ogun State, Nigeria. 
linear model in the presence of autocorrelation (Johnston, 1984; Chartterjee et.al, 2000; Maddala, 2002). To compensate for this lost of efficiency, Cochrane and Orcutt (1949) suggested a transformation of the regression model via the generalized least square (GLS) estimator. Chipman (1979), Kramer (1980), Kleiber (2001) and many others did observe that the efficiency of these estimators depends on the structure

of the regressors that are used. Rao and Griliches (1969) did one of the earliest Monte Carlo studies on the performances of some of these estimators with autoregressive stochastic regressor. They observed that the OLS estimator is only more efficient than any of the GLS estimators considered when $|\rho|<0.3$; and that the performances of the GLS estimators are not far apart. Park and Mitchell (1980) observed that when regressors are trended, the estimator that uses the $P$ transformation (Paris - Winstern) is more efficient than the one that uses the $Q$ transformation (Cochrane - Orcutt) and that the latter should even be avoided since it is less efficient than the OLS estimator.

More recently, Nwabueze (2005) examined the performance of some of these estimators with exponential independent variable. His result, among other things, show that the OLS estimator compares favorably with the Maximum Likelihood (ML) and Maximum Likelihood Grid (MLGD) estimators for small value of $\rho$ but it appears to be superior to Cochrane - Orcutt (CORC) and the Hidreth and Lu (HILU) especially when $\rho$ is large. Some other recent works that are done with different specification of regressors include that of lyaniwura and Nwabuwze (2004a), lyaniwura and Nwabuwze (2004b) and Olaomi and Iyaniwura (2006).

Consequently, this paper compares the performances of some of these estimators when normally distributed regressors are fixed in repeated sampling with when they are stochastic.

\section{METHODOLOGY}

Consider the GLS model with AR (1) of the form

$$
y_{t}=\beta_{0}+\beta_{1} x_{1 t}+\beta_{2} x_{2 t}+u_{t}
$$

where $u_{t}=\rho u_{t-1}+\varepsilon_{t}$

$$
|\rho|<1 \quad t=1,2, \ldots, n \quad \varepsilon_{t} \sim N\left(0, \sigma^{2}\right)
$$

Its parameter estimations can be done using the OLS and the (feasible) GLS estimators. Thus, the performances of the OLS estimator and the following feasible GLS estimators are studied: CORC, HILU, ML and the MLGD estimators. Monte Carlo experiments were performed 120 times for three sample sizes $(n=20,40,80)$ and four levels of autocorrelation ( $\rho=0.4,0.8,0.9,0.99$ ) with both fixed and stochastic regressors that are normally distributed. At a particular specification of $\mathrm{n}$ and $\rho$ (a scenario), the first replication was obtained by generating $e_{t} \sim N(0,1)$ and hence $u_{t}$. Assuming the process start from infinite past and continue to operate, the initial value of $U$ (i.e $u_{1}$ ) was thus drawn from a normal population with mean zero and variance $\frac{1}{1-\rho_{1}^{2}}$.

$$
\text { Hence } \begin{gathered}
u_{1}=\frac{\varepsilon_{1}}{\sqrt{1-\rho_{1}^{2}}} \\
u_{t}=\rho_{1} u_{t-1}+\varepsilon_{t} \mathrm{t}=2,3, \ldots, \mathrm{n}
\end{gathered}
$$

Furthermore, $x_{1 t} \sim N(0,1)$ and $x_{2 t} \sim N(0,1)$ were generated. Hence, the values of $y_{t}$ in equation (1) were also calculated by setting the true regression coefficients as $\beta_{0}=\beta_{1}=\beta_{2}=1$. This process continued until all replications in this scenario were obtained. Another scenario then started until all the scenarios were completed. The only difference in these procedures with stochastic regressors is that at each replication the $x_{1 t} \sim N(0,1)$ and $x_{2 t} \sim N(0,1)$ were newly generated.

Evaluation and comparison of estimators were examined using the finite sampling properties of estimators which include bias (B), and variance (Var) and the mean squared error (MSE) criteria. Mathematically, for any estimator $\hat{\beta}_{i}$ of $\beta_{i}$ of model (3)

$$
\begin{gathered}
\hat{\beta}_{i}=\frac{1}{120} \sum_{j=1}^{120} \hat{\beta}_{i j} \\
B\left(\begin{array}{c}
\wedge \\
\beta_{i}
\end{array}\right)=\frac{1}{120} \sum_{j=1}^{120}\left(\hat{\beta}_{i j}-\beta_{i}\right)=\hat{\beta}_{i}-\beta_{i} \\
\operatorname{Var}\left(\hat{\beta_{i}}\right)=\frac{1}{120} \sum_{j=1}^{120}\left(\hat{\beta}_{i j}-\hat{\beta}_{i}\right)^{2} \\
\operatorname{MSE}\left(\hat{\beta_{i}}\right)=\frac{1}{120} \sum_{j=1}^{120}\left(\hat{\beta_{i j}}-\beta_{i}\right)^{2}=\operatorname{Var}\left(\hat{\beta_{i}}\right)+\left[B\left(\hat{\beta_{i}}\right)\right]^{2} \\
\text { for i }=0,1,2 \text { and } \mathrm{j}=1,2, \ldots, 120 .
\end{gathered}
$$

For each of the estimation methods, a computer program was written using TSP software to estimate all the model parameters and to evaluate the criteria. Often times, preference of estimators are based on bias (closest to zero), minimum variance and minimum (root) mean squared error. In this study, we utilized the criteria of sum of bias (SBIAS), sum of variance (SVAR), and the root mean squared error (SRMSE) of the estimated model parameters to compare the performances of the estimators. This approach has also been used by lyaniwura and Nwabueze (2004a), lyaniwura and Nwabueze (2004b), Nwabueze (2005), Olaomi and Iyaniwura (2006) and some others.

Consider an estimator $\hat{\beta}()=.\left(\hat{\beta}_{0}, \hat{\beta}_{1}, \hat{\beta}_{2}\right)$, then

SBIAS of $\hat{\beta}()=.|B B 0|+|B B 1|+|B B 2|$

SVAR of $\hat{\beta}()=.V A R B 0+V A R B 1+V A R B 2$

SRMSE of $\hat{\beta}()=.R M S E B 0+R M S E B 1+R M S E B 2$

The efficiency of the estimators was further examined using the sum of SRMSE. An estimator with the smallest SRMSE is most efficient whereas if two estimators are nearly equal in terms of their SRMSE, they are simply said to be more efficient.

\section{SIMULATION RESULTS AND DISCUSSION}

The summary of the performances of the estimators on the basis of the sum of the criteria is given in table 1, 2 and 
3 in tne appenaix. AIso, tne summary or tne most more efficient estimator(s) is shown in table 4 in the appendix. However, the estimated criteria of the model parameters for $n$ $=20$ for both fixed and stochastic regressors are given in table $5,6,7,8,9$ and 10 in the appendix while for all other sample sizes, see Ayinde (2006).

From the tables, it is observed that at each level of autocorrelation the estimated value of the criteria with stochastic regressor are much lesser than that of the fixed regressor for all the estimators except CORC when the sample size is small $(n=20)$ where the CORC estimator has estimated value of the criteria greater with stochastic regressor than the fixed regressor especially when the level of autocorrelation is very high $(\rho=0.9)$. Also, with both fixed and stochastic regressor, as $\rho$ increases the estimated criteria of the estimators in all the sample sizes increase. Asymptotically, it is also observed that the estimated value of the criteria reduce at each level of autocorrelation.

Furthermore, in terms of the efficiency measured from table 1, 2 and 3 using the sum of root mean squared error of the estimated parameters, the summary of the results is shown in table 5 in the appendix.

From table 4, it can be seen that when the sample size is small $(\mathrm{n}=20)$ and the level of autocorrelation is both low $(\rho=0.4)$ and high $(\rho=0.8)$ the $\mathrm{ML} / \mathrm{MLGD}$ estimator is more efficient; and that at the other levels of autocorrelation the HILU estimator is most efficient. When the sample size is moderate $(n=40)$, the results are essentially the same with when the sample size is small $(n=20)$ except that the CORC estimator is now more efficient at high level of autocorrelation $(\rho=0.8)$; and also it competes with the HILU estimator when autocorrelation is very high $(\rho=0.9)$ under fixed regressors. Furthermore, when the sample size is large $(n=80)$ the results are the same with when the sample size is moderate $(n=40)$ except that when the autocorrelation level is high $(\rho=0.8)$ under stochastic regressors, the ML / MLGD estimators are more efficient; and under fixed regressors when autocorrelation is $\operatorname{low}(\rho=0.4)$ the CORC / HILU estimators are more efficient. Moreover, when the autocorrelation level is very high $(\rho=0.9)$ under stochastic regressors the CORC estimator also competes with HILU estimator.

Comparatively from table 5 , it is observed that the same estimator(s) that is more efficient with fixed regressors is also more efficient with stochastic regressors except when the sample size is large $(n=80)$ and the level of autocorrelation is either low $(\rho=0.4)$ or high $(\rho=0.8)$. At these instances, the CORC / HILU estimator is more efficient with fixed regressors while the ML / MLGD estimator is more efficient with stochastic regressors.

\section{CONCLUSION}

The performances of estimators of linear model in the presence of autocorrelated error terms with stochastic regressors are often much lesser than that of the fixed regressors on the basis of finite properties of estimator criteria. However, the estimators' performances in terms of their efficiency are much alike except when the sample size is large and the level of autocorrelation moderately high.

\section{REFERENCES}

Aitken, A. C., 1935. On Least Squares and Linear combinations of observations. Proceedings of the Royal Statistical Society. Edinburgh, 55: 42 - 48.

Ayinde, K., 2006. A Study of Robustness of Some methods of Parameter Estimation in Regression Model to of Ilorin, Nigeria.

Beach, C.M. and Mackinnon, J. S., 1978. A Maximum Likelihood Procedure for Regression with autocorrelated errors. Econometrica 46(1): 51 57.

Chipman, J. S., 1979. Efficiency of Least Squares Estimation of Linear Trend when residuals are autocorrelated Econometrica 47: 115 - 127.

Cochrane, D. and Orcutt, G. H., 1949. Application of Least Square to Relationship Containing Autocorrelated Error Terms. Journal of the American Statistical Association 44: $32-61$.

Durbin, J., 1960. Estimation of Parameters in Time - Series Regression Models. Journal of the Royal Statistical Society, B. 22: 139 - 153.

Fomby, T. B., Hill, R. C. and Johnson, S. R., 1984. Advanced Econometric Methods. Springer - Verlag, New York Berlin Heidelberg London Paris Tokyo.

Graybill, F. A., 1961. An introduction to Linear Statistical Models. New York. McGraw - Hill.

Hildreth, C. and Lu, J. Y., 1960. Demand Relationships with Autocorrelated Disturbances. Michigan State University. Agricultural Experiment Statistical Bulletin 276, East Lansing, Michigan.

Iyaniwura, J. O. and Nwabueze, J. C., 2004a. Estimators of Linear Model with Autocorrelated Error Terms and Trended Independent Variable. Journal of Nigerian Statistical Association, Volume 17: 20 - 28.

Iyaniwura, J.O. and Nwabueze, J.C., 2004b. Estimating the Autocorrelated Error Model with GNP Data. Journal of Nigerian Statistical Association, Volume 17: 2912.

Olaomi, J.O. and Iyaniwura, J. O., 2006. Efficiency of GLS estimators in Linear Regression Model with Autocorrelated Error Terms which are Also correlated with Regressor. An International Journal of Biological and Physical Science (Science Focus. 11(1): $129-133$

Kleiber, C., 2001. Finite Sample Efficiency of OLS in Linear Regression Models with Long-Memory Disturbances. Economic Letters, 72: 131 - 136.

Kramer, W., 1980. Finite Sample Efficiency of Ordinary Least Squares in the Linear Regression Model with Autocorrelated Errors. Journal of the American Statistical Association. 81: 150 - 154.

Maddala G. S., 2002. Introduction to Econometrics. John Wiley and sons Lt, England. $3^{\text {rd }}$ Edition.

Neter, J. and Wasserman, W., 1974. Applied Linear Model. Richard D. Irwin, Inc.

Nwabueze, J.C., 2005. Performances of estimators of linear auto - correlated model with exponential independent variable. Journal of Nigerian Association of Mathematical Physics, Volume 9: $385-388$.

Park, R.E. and Mitchell, B.M., 1980. Estimating the Autocorrelated Error Model with Trended Data. Journal of Econometrics, 13: $185-201$. 
Prais, S. J. and Winstein, C. B., 1954. Trend Estimators and Serial Correlation. Unpublished Cowles Commission, Discussion Paper, Chicago.

Rao, P. and Griliches, Z., 1969. Small Sample Properties of Several Two-Stage Regression Methods in the context of Autocorrelation Errors. Journal of the American Statistical Association, 64: 251 - 272.

Sampson, A. P., 1974. A tale of two regressions. Journal of the American Statistical Association, 69: 682-689.

Theil, H., 1971. Principle of Econometrics. New York, John Wiley and sons.
Thornton, D.L, 1982. The appropriate autocorrelation transformation when the autocorrelation process has a finite past. Federal Reserve Bank of St. Louis Working Paper, 82 - 002.

TSP, 2005. Users Guide and Reference Manual. Time Series Processor. New York.

White, K. J., 1978. A General Computer Program for Econometric Methods-SHAZAM. Econometrica, 46: 239-240.

\section{APPENDIX:}

TABLE 1: Sum of the estimated criteria of the model parameters when $n=20$

\begin{tabular}{|c|c|c|c|c|c|c|c|}
\hline \multirow[t]{2}{*}{$\rho$} & \multirow[t]{2}{*}{ Estimator } & \multicolumn{2}{|c|}{ Sum of Biases } & \multicolumn{2}{|c|}{ Sum of Variances } & \multicolumn{2}{|c|}{$\begin{array}{l}\text { Sum of Root Mean } \\
\text { Squared Error }\end{array}$} \\
\hline & & Fixed & Stochastic & Fixed & Stochastic & Fixed & Stochastic \\
\hline \multirow{5}{*}{0.4} & OLS & .238270 & .038352 & 5.775682 & .166334 & 2.984762 & .656117 \\
\hline & CORC & .204020 & .047266 & 5.798128 & .175474 & 2.941968 & .709747 \\
\hline & HILU & .205320 & .050004 & 5.789748 & .180270 & 2.940498 & .724220 \\
\hline & $\mathrm{ML}$ & .234019 & .039626 & 5.745688 & .146264 & 2.924859 & .630946 \\
\hline & MLGD & .233891 & .037927 & 5.744684 & .145209 & 2.924527 & .627941 \\
\hline \multirow{5}{*}{0.8} & OLS & .266471 & .056133 & 6.153009 & .363389 & 3.263503 & 1.043938 \\
\hline & CORC & .039216 & .125415 & 7.181041 & .653176 & 3.157918 & 1.224348 \\
\hline & HILU & .186433 & .073298 & 5.956764 & .388445 & 2.917448 & .993977 \\
\hline & $\mathrm{ML}$ & .216507 & .027413 & 5.834747 & .205884 & 2.881965 & .770204 \\
\hline & MLGD & .218063 & .041021 & 5.846870 & .187885 & 2.885169 & .734114 \\
\hline \multirow{5}{*}{0.9} & OLS & .281376 & .067919 & 6.984565 & .979242 & 3.502758 & 1.607896 \\
\hline & CORC & .124364 & .839709 & 8.636493 & 78.423288 & 3.392219 & 9.314332 \\
\hline & HILU & .239270 & .068723 & 5.998021 & .451160 & 2.908556 & 1.029051 \\
\hline & ML & .226088 & .047919 & 6.385660 & .657157 & 2.973699 & 1.152051 \\
\hline & MLGD & .229164 & .053341 & 6.396638 & .661847 & 2.977209 & 1.155442 \\
\hline \multirow{5}{*}{0.99} & OLS & .283102 & .080187 & 38.345911 & 31.889861 & 7.094184 & 6.389459 \\
\hline & CORC & .662101 & .406078 & 43.485020 & 29.826651 & 7.067019 & 5.859107 \\
\hline & HILU & .266153 & .036515 & 30.009107 & 24.180186 & 5.913894 & 5.301591 \\
\hline & $\mathrm{ML}$ & .252761 & .074358 & 36.939508 & 30.899853 & 6.499316 & 5.932051 \\
\hline & MLGD & .249847 & .072162 & 36.939690 & 30.989355 & 6.500296 & 5.940578 \\
\hline
\end{tabular}

TABLE 2: Sum of the estimated criteria of the model parameters when $n=40$

\begin{tabular}{|c|c|c|c|c|c|c|c|}
\hline \multirow[t]{2}{*}{$\rho$} & \multirow{2}{*}{$\begin{array}{l}\text { Estimat } \\
\text { or }\end{array}$} & \multicolumn{2}{|c|}{ Sum of Biases } & \multicolumn{2}{|c|}{ Sum of Variances } & \multicolumn{2}{|c|}{$\begin{array}{c}\text { Sum of Root Mean Squared } \\
\text { Error }\end{array}$} \\
\hline & & Fixed & Stochastic & Fixed & Stochastic & Fixed & Stochastic \\
\hline \multirow{5}{*}{0.4} & OLS & .247033 & .019700 & 5.667157 & .068791 & 2.776989 & .411387 \\
\hline & CORC & .240610 & .024383 & 5.639120 & .053530 & 2.721794 & .379146 \\
\hline & HILU & .240863 & .024651 & 5.639213 & .052998 & 2.721970 & .377509 \\
\hline & $\mathrm{ML}$ & .239337 & .019273 & 5.646078 & .051251 & 2.720489 & .356836 \\
\hline & MLGD & .239800 & .018713 & 5.645847 & .050760 & 2.719910 & .355102 \\
\hline \multirow{5}{*}{0.8} & OLS & .279389 & .045788 & 5.821598 & .142195 & 2.999537 & .642281 \\
\hline & CORC & .225009 & .027036 & 5.651256 & .053457 & 2.675785 & .400956 \\
\hline & HILU & .225662 & .030867 & 5.650916 & .061062 & 2.675680 & .425629 \\
\hline & $\mathrm{ML}$ & .243775 & .021199 & 5.697144 & .060774 & 2.686009 & .424054 \\
\hline & MLGD & .245195 & .025081 & 5.700142 & .064486 & 2.687561 & .435030 \\
\hline \multirow{5}{*}{0.9} & OLS & .343057 & .086436 & 6.099065 & .354921 & 3.175278 & 1.010561 \\
\hline & CORC & .226712 & .027416 & 5.757145 & .134072 & 2.686770 & .566352 \\
\hline & HILU & .224773 & .027344 & 5.741233 & .107847 & 2.682936 & .522869 \\
\hline & $\mathrm{ML}$ & .276233 & .050594 & 5.921211 & .241752 & 2.724562 & .705002 \\
\hline & MLGD & .276014 & .049323 & 5.912873 & .231940 & 2.722778 & .694261 \\
\hline \multirow{5}{*}{0.99} & OLS & .760960 & .456681 & 29.513130 & 22.885019 & 6.301095 & 5.419174 \\
\hline & CORC & .511734 & .370717 & 159.821391 & 16.674900 & 12.926464 & 4.326374 \\
\hline & HILU & .501084 & .248016 & 22.810143 & 16.397971 & 5.073257 & 4.283857 \\
\hline & $\mathrm{ML}$ & .671973 & .423473 & 29.416089 & 22.913213 & 5.735637 & 5.032298 \\
\hline & MLGD & .665383 & .418786 & 29.144429 & 22.647244 & 5.709859 & 5.004314 \\
\hline
\end{tabular}


TABLE 3: Sum of the estimated criteria of the model parameters when $n=80$

\begin{tabular}{|c|c|c|c|c|c|c|c|}
\hline \multirow{3}{*}{$\rho$} & \multirow{2}{*}{ Estimator } & \multicolumn{2}{|c|}{ Sum of Biases } & \multicolumn{2}{c|}{ Sum of Variances } & \multicolumn{2}{c|}{$\begin{array}{c}\text { Sum of Root Mean } \\
\text { Squared Error }\end{array}$} \\
\cline { 3 - 7 } & & Fixed & Stochastic & Fixed & Stochastic & Fixed & Stochastic \\
\hline \multirow{4}{*}{0.4} & OLS & .250802 & .014099 & 5.636964 & .035007 & 2.684490 & .286915 \\
& CORC & .252363 & .017475 & 5.606360 & .025552 & 2.644359 & .254305 \\
& HILU & .252816 & .018032 & 5.608606 & .025365 & 2.645570 & .253706 \\
& ML & .252489 & .015358 & 5.627886 & .024661 & 2.649754 & .242854 \\
& MLGD & .252547 & .016199 & 5.628093 & .024715 & 2.649549 & .243370 \\
\hline \multirow{4}{*}{0.8} & OLS & .263009 & .037144 & 5.678403 & .085684 & 2.812667 & .475116 \\
& CORC & .240028 & .020998 & 5.576679 & .025315 & 2.611712 & .276711 \\
& HIU & .236382 & .024039 & 5.583061 & .026128 & 2.613064 & .281463 \\
& ML & .247864 & .013629 & 5.668202 & .024962 & 2.632163 & .274328 \\
& MLGD & .246783 & .012750 & 5.663375 & .025273 & 2.631302 & .276023 \\
\hline \multirow{5}{*}{0.9} & OLS & .265469 & .055593 & 5.730489 & .187614 & 2.916687 & .752543 \\
& CORC & .234145 & .028050 & 5.570998 & .061649 & 2.602313 & .392062 \\
& HILU & .215455 & .043050 & 5.581487 & .062544 & 2.603192 & .395977 \\
& ML & .259666 & .028731 & 5.800086 & .096059 & 2.652877 & .460007 \\
& MLGD & .254529 & .025007 & 5.782619 & .090554 & 2.648944 & .450261 \\
\hline \multirow{6}{*}{0.99} & OLS & .565490 & .414638 & 27.642830 & 21.321966 & 5.981096 & 5.290383 \\
& CORC & .419035 & .201564 & 16.338757 & 10.815012 & 4.289645 & 3.459889 \\
& HILU & .328511 & .270800 & 7.995106 & 8.112876 & 3.070842 & 3.025455 \\
& ML & .545399 & .368202 & 25.739784 & 19.552746 & 5.328961 & 4.602012 \\
& MLGD & .515371 & .346511 & 26.498670 & 20.258198 & 5.400255 & 4.679430 \\
\hline
\end{tabular}

TABLE 4: Summary of the more / most efficient estimator(s)

\begin{tabular}{|c|c|c|c|}
\hline \multirow{2}{*}{$\begin{array}{l}\text { Sample } \\
\text { size (n) }\end{array}$} & \multirow[b]{2}{*}{$\rho$} & \multicolumn{2}{|c|}{ Regressors } \\
\hline & & Fixed & Stochastic \\
\hline \multirow{4}{*}{20} & 0.4 & ML/MLGD & ML/MLGD \\
\hline & 0.8 & ML/MLGD & ML/MLGD \\
\hline & 0.9 & HILU & HILU \\
\hline & 0.99 & HILU & HILU \\
\hline \multirow{4}{*}{40} & 0.4 & ML/MLGD & ML/MLGD \\
\hline & 0.8 & CORC/HILU & CORC \\
\hline & 0.9 & CORC/HILU & HILU \\
\hline & 0.99 & HILU & HILU \\
\hline \multirow{4}{*}{80} & 0.4 & CORC/HILU & ML/MLGD \\
\hline & 0.8 & CORC/HILU & ML/MLGD \\
\hline & 0.9 & CORC/HILU & CORC/HILU \\
\hline & 0.99 & HILU & HILU \\
\hline
\end{tabular}

TABLE 5: Bias of $\beta$ with fixed regressor when $\mathrm{n}=20$ and $\mathrm{R}=120$.

\begin{tabular}{|c|c|c|c|c|c|}
\hline$\rho$ & Estimator & BB0 & BB1 & BB2 & SBIAS \\
\hline \multirow{4}{*}{0.4} & OLS & .218460 & -.006482 & -.013328 & .238270 \\
& CORC & .182840 & -.017640 & .003540 & .204020 \\
& HILU & .184910 & -.017215 & .003195 & .205320 \\
& ML & .212750 & -.019451 & -.001818 & .234019 \\
& MLGD & .213070 & -.018807 & -.002014 & .233891 \\
\hline \multirow{4}{*}{0.8} & OLS & .232580 & -.011302 & -.022589 & .266471 \\
& CORC & .038880 & .000311 & -.000026 & .039216 \\
& HILU & .176440 & .006256 & .003738 & .186433 \\
& ML & .208870 & .005978 & -.001659 & .216507 \\
& MLGD & .209900 & .006465 & -.001698 & .218063 \\
\hline \multirow{4}{*}{0.9} & OLS & .249260 & -.006339 & -.025777 & .281376 \\
& CORC & .118030 & .001118 & .005217 & .124364 \\
& HILU & .232320 & .000479 & .006471 & .239270 \\
& ML & .222830 & .001941 & -.001317 & .226088 \\
& MLGD & .225710 & .002104 & -.001351 & .229164 \\
\hline \multirow{6}{*}{0.99} & OLS & .251330 & .007021 & -.024751 & .283102 \\
& CORC & .644360 & .013240 & .004501 & .662101 \\
& HILU & .254380 & .011488 & -.000285 & .266153 \\
& ML & .238320 & .011103 & -.003338 & .252761 \\
& MLGD & .235750 & .011575 & -.002522 & .249847 \\
\hline
\end{tabular}


TABLE 6: Variance of $\beta$ with fixed regressor when $\mathrm{n}=20$ and $\mathrm{R}=120$.

\begin{tabular}{|c|c|c|c|c|c|}
\hline$\rho$ & Estimator & VB0 & VB1 & VB2 & SVAR \\
\hline \multirow{4}{*}{0.4} & OLS & 5.589455 & .097461 & .088765 & 5.775682 \\
& CORC & 5.641770 & .080830 & .075528 & 5.798128 \\
& HILU & 5.633298 & .080601 & .075849 & 5.789748 \\
& ML & 5.594737 & .078916 & .072035 & 5.745688 \\
& MLGD & 5.593811 & .078683 & .072190 & 5.744684 \\
\hline \multirow{4}{*}{0.8} & OLS & 5.793627 & .211262 & .148120 & 6.153009 \\
& CORC & 7.055148 & .058578 & .067315 & 7.181041 \\
& HILU & 5.833769 & .056623 & .066372 & 5.956764 \\
& ML & 5.718863 & .056167 & .059716 & 5.834747 \\
& MLGD & 5.730682 & .056453 & .059735 & 5.846870 \\
\hline \multirow{5}{*}{0.9} & OLS & 6.545189 & .268890 & .170486 & 6.984565 \\
& CORC & 8.525999 & .051646 & .058849 & 8.636493 \\
& HILU & 5.886957 & .052009 & .059055 & 5.998021 \\
& ML & 6.280947 & .051563 & .053150 & 6.385660 \\
& MLGD & 6.291375 & .051390 & .053873 & 6.396638 \\
\hline \multirow{5}{*}{0.99} & OLS & 37.905503 & .273501 & .166907 & 38.345911 \\
& CORC & 43.384100 & .044421 & .056499 & 43.485020 \\
& HILU & 29.912821 & .043909 & .052377 & 30.009107 \\
& ML & 36.849624 & .043389 & .046496 & 36.939508 \\
& MLGD & 36.849352 & .043932 & .046406 & 36.939690 \\
\hline
\end{tabular}

TABLE 7: Root mean squared of $\beta$ with fixed regressor when $\mathrm{n}=20$ and $\mathrm{R}=120$.

\begin{tabular}{|c|c|c|c|c|c|}
\hline$\rho$ & Estimator & RMB0 & RMB1 & RMB2 & SRMSE \\
\hline \multirow{4}{*}{0.4} & OLS & 2.374275 & .312255 & .298233 & 2.984762 \\
& CORC & 2.382268 & .284853 & .274847 & 2.941968 \\
& HILU & 2.380649 & .284424 & .275425 & 2.940498 \\
& ML & 2.374868 & .281592 & .268399 & 2.924859 \\
& MLGD & 2.374702 & .281135 & .268689 & 2.924527 \\
\hline \multirow{4}{*}{0.8} & OLS & 2.418206 & .459772 & .385526 & 3.263503 \\
& CORC & 2.656437 & .242029 & .259451 & 3.157918 \\
& HILU & 2.421756 & .238038 & .257655 & 2.917448 \\
& ML & 2.400519 & .237072 & .244375 & 2.881965 \\
& MLGD & 2.403069 & .237687 & .244414 & 2.885169 \\
\hline \multirow{5}{*}{0.9} & OLS & 2.570471 & .518585 & .413703 & 3.502758 \\
& CORC & 2.922316 & .227260 & .242644 & 3.392219 \\
& HILU & 2.437402 & .228055 & .243099 & 2.908556 \\
& ML & 2.516068 & .227084 & .230547 & 2.973699 \\
& MLGD & 2.518396 & .226702 & .232110 & 2.977209 \\
\hline \multirow{5}{*}{0.99} & OLS & 6.161872 & .523020 & .409292 & 7.094184 \\
& CORC & 6.618104 & .211178 & .237737 & 7.067019 \\
& HILU & 5.475174 & .209859 & .228860 & 5.913894 \\
& ML & 6.075065 & .208595 & .215655 & 6.499316 \\
& MLGD & 6.074943 & .209919 & .215434 & 6.500296 \\
\hline
\end{tabular}

TABLE 8: Bias of $\beta$ with stochastic regressors when $\mathrm{n}=20$ and $\mathrm{R}=120$

\begin{tabular}{|c|c|c|c|c|c|}
\hline$\rho$ & Estimator & BB0 & BB1 & BB2 & SBIAS \\
\hline \multirow{4}{*}{0.4} & OLS & .007344 & -.029209 & .001799 & .038352 \\
& CORC & -.014375 & -.018075 & -.014816 & .047266 \\
& HILU & -.017108 & -.018296 & -.014600 & .050004 \\
& ML & .008449 & -.024703 & -.006475 & .039626 \\
& MLGD & .007297 & -.024030 & -.006600 & .037927 \\
\hline \multirow{4}{*}{0.8} & OLS & .020933 & -.018791 & -.016409 & .056133 \\
& CORC & -.093311 & -.010060 & -.022044 & .125415 \\
& HILU & -.031898 & -.008305 & -.033095 & .073298 \\
& ML & .004288 & -.012049 & -.011076 & .027413 \\
& MLGD & .009819 & -.011101 & -.020101 & .041021 \\
\hline \multirow{5}{*}{0.9} & OLS & .038194 & -.004922 & -.024803 & .067919 \\
& CORC & .806880 & -.003197 & -.029632 & .839709 \\
& HILU & .035737 & -.003658 & -.029328 & .068723 \\
& ML & .023303 & -.005196 & -.019420 & .047919 \\
& MLGD & .028056 & -.005956 & -.019329 & .053341 \\
\hline \multirow{5}{*}{0.99} & OLS & .055476 & .004100 & -.020611 & .080187 \\
& CORC & .377050 & -.003457 & -.025571 & .406078 \\
& HILU & .006200 & -.004365 & -.025951 & .036515 \\
& ML & .049108 & -.003146 & -.022104 & .074358 \\
& & .047197 & -.002862 & -.022103 & .072162 \\
\hline
\end{tabular}


TABLE 9: Variance of $\beta$ with stochastic regressors when $\mathrm{n}=20$ and $\mathrm{R}=120$

\begin{tabular}{|c|c|c|c|c|c|}
\hline$\rho$ & Estimator & VB0 & VB1 & VB2 & SVAR \\
\hline \multirow{5}{*}{0.4} & OLS & .008582 & .076754 & .080999 & .166334 \\
& CORC & .026956 & .064870 & .083647 & .175474 \\
& HILU & .031960 & .065082 & .083228 & .180270 \\
& ML & .012941 & .059106 & .074218 & .146264 \\
& MLGD & .012628 & .058566 & .074015 & .145209 \\
\hline \multirow{4}{*}{0.8} & OLS & .104922 & .119527 & .138941 & .363389 \\
& CORC & .531943 & .044476 & .076757 & .653176 \\
& HILU & .281693 & .043200 & .063553 & .388445 \\
& ML & .107592 & .040263 & .058029 & .205884 \\
& MLGD & .102914 & .039077 & .045895 & .187885 \\
\hline \multirow{5}{*}{0.9} & OLS & .667371 & .145056 & .166815 & .979242 \\
& CORC & 78.332465 & .038960 & .051864 & 78.423288 \\
& HILU & .359883 & .039142 & .052136 & .451160 \\
& ML & .581877 & .035551 & .039729 & .657157 \\
& MLGD & .586503 & .035784 & .039560 & .661847 \\
\hline \multirow{6}{*}{0.99} & OLS & 31.594642 & .135223 & .159995 & 31.889861 \\
& CORC & 29.750583 & .036910 & .039158 & 29.826651 \\
& HILU & 24.104022 & .037053 & .039112 & 24.180186 \\
& ML & 30.828288 & .034149 & .037415 & 30.899853 \\
& MLGD & 30.917592 & .034183 & .037579 & 30.989355 \\
\hline
\end{tabular}

TABLE 10: Root mean squared error of $\beta$ with stochastic regressors when $\mathrm{n}=20$ and $\mathrm{R}=120$

\begin{tabular}{|c|c|c|c|c|c|}
\hline$\rho$ & Estimator & RMB0 & RMB1 & RMB2 & SRMSE \\
\hline \multirow{5}{*}{0.4} & OLS & .092928 & .278580 & .284609 & .656117 \\
& CORC & .164812 & .255337 & .289598 & .709747 \\
& HILU & .179591 & .255767 & .288862 & .724220 \\
& ML & .114070 & .244369 & .272507 & .630946 \\
& MLGD & .112610 & .243193 & .272138 & .627941 \\
\hline \multirow{5}{*}{0.8} & OLS & .324592 & .346237 & .373109 & 1.043938 \\
& CORC & .735289 & .211133 & .277926 & 1.224348 \\
& HILU & .531705 & .208012 & .254260 & .993977 \\
& ML & .328040 & .201017 & .241147 & .770204 \\
& MLGD & .320952 & .197990 & .215172 & .734114 \\
\hline \multirow{5}{*}{0.9} & OLS & .817820 & .380894 & .409182 & 1.607896 \\
& CORC & 8.887267 & .197408 & .229656 & 9.314332 \\
& HILU & .600966 & .197876 & .230209 & 1.029051 \\
& ML & .763164 & .188621 & .200265 & 1.152051 \\
& MLGD & .766348 & .189259 & .199835 & 1.155442 \\
\hline \multirow{6}{*}{0.99} & OLS & 5.621185 & .367750 & .400525 & 6.389459 \\
& CORC & 5.467426 & .192151 & .199529 & 5.859107 \\
& HILU & 4.909589 & .192541 & .199462 & 5.301591 \\
& ML & 5.552540 & .184822 & .194689 & 5.932051 \\
& MLGD & 5.560559 & .184908 & .195110 & 5.940578 \\
\hline
\end{tabular}

